\title{
Numerical and Experiment Study of the Correlation between Skin Blood Flow Oscillation and Radial Pulse at Wrist
}

\author{
Shuang Tian ${ }^{1}$, Tianxi $\mathrm{Chi}^{1}$, Yang Liu ${ }^{1}$, Zhongdi Su${ }^{2}$ \\ ${ }^{1}$ Department of Mechanical Engineering, The Hong Kong Polytechnic University \\ Hung Hom, Kowloon, Hong Kong, PRC \\ mmyliu@polyu.edu.hk \\ ${ }^{2}$ College of Metrology and Measurement Engineering, China Jiliang University \\ Hang Zhou, China
}

\section{Extended Abstract}

The radial stretch of the ascending aorta brought about by left ventricular ejection initiates a pressure wave that is propagated down the aorta and its branches. To determine heart rate, one feels the pulse in the radial artery at the wrist. What is felt is not the blood pulsing through the arteries but a shock wave that travels along the walls of the arteries as the heart contracts. The typical heart rate is $\sim 1 \mathrm{~Hz}$. However in our preliminary measurement of blood flow oscillation in radial artery at the wrist, we have found that the power spectral density (PSD) at $0.001 \sim 0.1 \mathrm{~Hz}$ is much higher than that at heart rate, indicating that low frequency flow oscillations play a dominant role in radial pulse pattern. The spectral intervals of blood flow oscillation are related to cardiac, respiratory, endothelial and myogenic activities, in which $\sim 1 \mathrm{~Hz}$ is related to cardiac activity, $\sim 0.3 \mathrm{~Hz}$ is related to respiratory activity, and $0.001 \sim 0.2 \mathrm{~Hz}$ is related to endothelial and myogenic activities [1]. Vasomotion is spontaneous time-dependent contraction and relaxation of micro arteries which is an intrinsic phenomenon related to endothelial and myogenic activities [2]. Different control mechanisms modulate diameter of arterioles which results in blood flow oscillations, the so called flowmotion that can be measured by laser-Doppler flowmetry (LDF).

There is evidence that microcirculation may mirror the vascular function of other parts of the body and the microvascular abnormalities may initiate the pathogenesis sequence in some diseases [1]. Moreover, the traditional Chinese physicians could decide the diagnosis entirely based on the radial pulse palpation at wrist. The dominant PSD of vasomotion indicates that microcirculation related pressure wave travels upstream back to radial artery, and the radial pulse pattern would reflect the status of flowmotion in microcirculation. We argue that what the Chinese physicians palpated is actually the status of microcirculation. Thus, the thorough understanding of interaction between vasomotion related and cardiac activity related pressure waves would enable us to develop novel diagnostic technique. Therefore, the experimental and numerical studies are carried out to understand the interaction between vasomotion and cardiac rhythm in radial artery at wrist, which is an attractive fluid-structure interaction research topic in itself.

The experimental study is carried out using LDF measurement in human subjects and numerical study is conducted using Computational Fluid Dynamics (CFD). It follows that the objective of the proposed project is to experimentally and numerically study the effect of vasomotion on the pulse pattern at wrist with emphasis placed on the coherence between vasomotion in microcirculation and flowmotion at wrist and mechanism of pressure wave travelling along arterial walls.

\section{Acknowledgement}

Supports given by the Research Grants Council of the Government of the HKSAR under grant No. PolyU 5202/13E and the Hong Kong Polytechnic University under grant No. G-YL41 are gratefully acknowledged.

\section{References}

[1] M. Rossi, A. Carpi., C. Di Maria, F. Galetta, and G. Santoro, "Spectral analysis of laser Doppler skin blood flow oscillations in human essential arterial hypertension," Microvascular research, vol. 72, pp. 34-41, 2006.

[2] H. Nilsson and C. Aalkjær, "Vasomotion: mechanisms and physiological importance," Mol Interv, vol. 3, pp. 79-89, 2003. 